\title{
A violência doméstica contra idosos nas áreas de abrangência do Programa Saúde da Família de Niterói (RJ, Brasil)
}

\author{
The domestic violence against the elderly \\ within the Family Health Program of Niterói (RJ, Brazil)
}

Paulo Cavalcante Apratto Júnior ${ }^{1}$

${ }^{1}$ Fundação M unicipal de Saúdede Niterói (RJ). Av. Ernani do Amaral Peixoto 169, Centro. 24020-070 Niterói RJ.

aprattoporto@terra.com.br
Abstract This article investigates the magnitude and characteristics of violence against the eldery by trusted people at Ilha da Conceição, N iterói RJ registered at the Family Health Program. A domestic survey interviewed 343 individuals with 60 years or more, sel ected by a simple random sample. To identify the violence it was used the Conflict Tactics Scales. Information about identification, demographics and socio-economics characteristics were obtained using the $\mathrm{N}$ ational $\mathrm{H}$ ealth Interview Survey. The M ini-M ental State Examination was used to evaluate mental health. In order to evaluate the functional capacity, the Health Assessment Questionnairewas used. In cases of alcohol suspicion among men, it was used the instrument CAGE. To female elder or caregivers, the TWEAK instrument was used. $43 \%$ reported at least oneepisode of psychological violence. Physical violence was reported by $9,6 \%$ of the interviewed, $6,1 \%$ reported serious physical violence in this period. The prevalence of different modalities of violence was higher among the youngest individuals, with higher scholarity, among those who have one of the pathologies that characterize the elderly as having a vulnerability (depression and / or urinary incontinence / fecal and / or diabetes and / or rheumatism) and those living with the greatest number of individuals.

Key words Domestic violence, Prevalence, Aging
Resumo Este artigo investiga a magnitude e as características da violência contra idosos no domicílio por pessoas de confiança no bairro da IIha da Concei ção, em Niterói (RJ), adscritos ao Programa Saúdeda Família. Através de um inquérito domiciliar, foram entrevistados 343 indivíduos com 60 anos ou mais, selecionados por amostragem aleatória simples. Para identificação de violência usouse a Escala Tática de Conflitos. As informações sobre identificação, características demográficas e socioeconômicas foram obtidas pela Pesquisa Nacional por Amostra de Domicílio. Avaliou-se a saúde mental pelo M iniexame do Estado M ental. Para avaliação da capacidade funcional, usou-se o H ealth Assessment Q uestionnaire. Quanto à suspeita de uso de álcool pelos homens, utilizou-se 0 instrumento CAGE; para a idosa ou cuidadora, o instrumento TWEAK. 43\% relataram pelo menos um episódio de violência psicológica. A violência física foi relatada por 9,6\% dosentrevistados; $6,1 \%$ referiram ocorrência de violência física grave nesseperíodo. A prevalência das diversas modalidades de violência foi maior entre os mais novos, com maior escolaridade, entre os que apresentam uma das patologias que caracterizam o idoso como tendo uma vulnerabilidade (depressão e/ ou incontinência urinária/fecal e/ou diabetes e/ou reumatismo) e entre os que moram com maior número de indivíduos.

Palavras-chave Violência doméstica, Prevalência, Envelhecimento 
Introdução

0 envelhecimento populacional no mundo éum dos maiores desafios contemporâneos da Saúde Pública. Essefenômeno ocorreu inicialmenteem países desenvolvidos, mas, recentemente, os países em desenvolvimento mostram o envelhecimento da população de forma mais acentuada ${ }^{1}$. No Brasil, o número de idosos passou de três milhões, em 1960, para setemilhões em 1975 e 14 milhões em 2002. Estima-se que alcançará 34 milhões em 2020, levando o país ao sexto lugar no ranking mundial de países com maior número de pessoas nessa faixa etária². Muitas são as dificuldades enfrentadas pelos idosos, algumas decorrentes da fragilidade e da vulnerabilidade fisi ológica dessa faixa etária, que os tornam vítimas em potencial de várias mazelas sociais, dentre as quais a crescente violência observada em nossos dias ${ }^{3}$.

Inicialmente, a violência contra os idosos era vista como uma questão familiar, permanecendo reservada e escondida até a metade do século $X X$. Representa, hoje, um grande desafio para o setor de saúde, atingindo todas as classes sociais e provocando, além de óbitos, traumas físicos e emocionais de grandemagnitude quecriam uma demanda por serviços e programas de saúdemais adequados. Por essa razão, é fundamental que os profissionais da área coloquem em pauta esse problema antigo, porém de baixa visibilidade, enfocando-o como prioridade na agenda de diagnóstico situacional e estabelecendo políticas para seu enfrentamento ${ }^{4}$. Dentre as várias formas de violência queacometem a população idosa, destacam-se os maus-tratos e a negligência cometidos no âmbito familiar e institucional merecendo, portanto, maior atenção de toda a sociedadecivil.

A violência éum problema mundial, comple xo, enraizado em dimensões culturais, que possibilita diferentes abordagens e definições, o que traz dificuldade para estudos comparativos e visões gl obais sobre sua magnitude ${ }^{5}$. A multiplicidade e a falta de integração das fontes de informação e as altas taxas de sub-registro também são desafios a serem superados visando a estimativas mais fidedignas ${ }^{6-8}$. A pesar dessas dificuldades, estudos internacionais têm sugerido quea violência contra o idoso tenha prevalências mais al tas do que muitas patologias alvos de programas de controle em todo o mundo,6-8.

No Brasil, ainda não se tem ideia da prevalência do problema. No entanto, as características da sociedade brasileira atual, tais como as sérias dificuldades socioeconômicas para um grande segmento populacional, o preconceito contra o envelhecimento e o culto à juventude, fatores reconhecidamente favorecedores da disseminação da violência, fazem crer queo problema seja bastante frequente, tornando de grande relevância no país a temática violência praticada contra os gerontes.

Grande parte das situações ainda sofre uma invisi bilidade de origem social. D estaca-se nessa invisi bilidade a difusão da ideia de que a violência é um problema privado, que só pode ser resolvido pelos envolvidos. Ademais, as escolas formadoras de profissionais da saúde ainda não os preparam para o manejo de casos de violência, contribuindo para sua não detecção. Como muitas vezes a violência é cometida por pessoas de sua confiança, o idoso não se dispõe a relatar facilmente os episódios de vitimização que sofre, dificultando ainda mais a identificação da situação. Além disso, a falta de instrumentos para detecção, bem como de um arsenal resolutivo para o enfrentamento do problema, faz com que os profissionais de saúde compactuem com essa invisibilidade ${ }^{9-11}$.

A Organização Pan-Americana da Saúdeconsidera que o setor saúde deve ajudar a buscar soluções eaplicações de medidas preventivas ede controle de todas as formas de violência nesse grupo populacional. N esse contexto, a estratégia Saúde da Família pode ter um enorme potencial para construir estratégias de prevenção, detecção precoce e acompanhamento de famílias em situação de violência. Os profissionais que estão inseridos nas comunidades, empenhados em propostas de educação em saúde com o objetivo de contribuir para a transformação social, podem ser efetivos agentes para o conhecimento ea intervenção nessa problemática da violência praticada contra idosos.

Infelizmente, a escassez de estudos sobre o tema impede que se tenha uma visão mais acurada da magnitude e caracterização do problema, dificultando um planejamento de ações efetivas para seu enfrentamento imediato.

Visando expandir o conhecimento sobre a situação no âmbito populacional, esteartigo tem como objetivo estimar a prevalência da violência psicológica eviolência física contra o idoso numa população de baixa renda assistida pelo Programa M édico de Família da cidade de Niterói (RJ). Como objetivo secundário, pretende-se avaliar a prevalência do evento em diferentes subgrupos populacionais de acordo com características sociodemográficas e de saúde do idoso e de sua 
família, contribuindo para um diagnóstico situacional que forneça subsídios para o enfrentamento do problema. Considerando a escassez de pesquisas na área e a participação cada vez maior dos programas de Saúde da Família no Sistema Único de Saúde (SUS) em todo o Brasil, pretende-se que os frutos deste estudo não se restrinjam às equipes que participaram da pesquisa, mas que possam servir como ponto de partida para o conjunto de profissionais de saúde comprometidos com a promoção da saúde desse grupo populacional, convergindo com a promoção do envelhecimento ativo, caracterizado pela experiência positiva de longevidade do indivíduo.

\section{Métodos}

\section{Contexto do estudo}

O bairro da Ilha da Conceição localiza-se no interior da baía de Guanabara. Esteéatualmente o principal polo metalúrgico naval e abriga também parte da indústria pesqueira de Niterói. De acordo com o censo realizado em 2006, a população do bairro compreende cerca de 6.500 habitantes. A presenta grande percentual de idosos, sendo um dos bairros do município que apresentam alto índice de envelhecimento ${ }^{12,13}$.

Para fins de cadastramento e acompanhamento das famílias pelas equipes do PM F, o bairro foi dividido em seis microáreas denominadas de setores $24,25,26,27,28$ e 29 . 0 processo de cadastramento incluiu até 250 famílias em cada setor, respeitando-se um número total máximo de 1.300 pessoas. Atualmente, o módulo contém seis equipes, o que permite uma cobertura de $100 \%$ do bairro pelo programa.

A territorialização é um dos pressupostos básicos do trabalho do PMF, por isso a llha da Conceiçãa foi dividido nas seis microáreas denominadas de setores $24,25,26,27,28$ e 29.0 cadastro contabilizou por setor até 250 famílias, totalizando no máximo 1.300 pessoas. Atualmente este módulo contém seis equipes (duplas de médicos eauxiliares de enfermagem), sendo todo 0 bairro adscrito e atendido pelo PM F.

\section{Desenho}

Trata-se de um estudo transversal de base populacional, desenvolvido na Estratégia Saúde da Família no M ódulo de Saúde Célia Sanchez, no bairro da I lha da Conceição, localizado na cidade de N iterói, Estado do Rio de Janeiro, Brasil.
População-alvo/fonte

A população-alvo do estudo é composta pelo conjunto de indivíduoscom 60 anosou mais, não institucionalizados, residentes em áreas urbanas cobertas pelo Programa Médico de Família do município deNiterói, Rio deJaneiro. Atualmente, - PMF atingiu $30 \%$ de cobertura de sua população e 150 mil pessoas já foram cadastradas em 33 áreas consideradas de desenvolvido risco social, com delineamentos na saúde e econômico.

Como população fonte, optou-se pela clientela de indivíduos dessa faixa etária, residentes e adscritos ao módulo do Programa Saúde da Família(PSF) no bairro da Ilha da Conceição (Niterói). Segundo dados do Sistema de Informação de Atenção Básica (SIAB), em setembro de 2006 esse grupo era composto por 754 indivíduos ${ }^{14}$.

Tamanho e estratégia de sel eção

da amostra do estudo

A constituição da amostra foi feita a partir de uma malha amostral formada por todos os idosos das microáreas 24, 25, 26, 27, 28 e 29, que compõem a área do Programa M édico de Família do M ódulo I lha da Conceição, através deuma seleção al eatória simples dos idosos nos diversos setores do bairro. 0 tamanho amostral foi definido considerando-se a população fonte (754), a prevalência esperada de violência ao idoso de $40 \%{ }^{15}$ e um erro de mais ou menos $5 \%{ }^{16}$. Com base nesses critérios, o tamanho amostral mínimo previsto foi de 327 gerontes moradores da Ilha da Conceição. Os critérios de inclusão no estudo foram: ter idade superior a 60 anos de idade na época da entrevista e concordar em participar da pesquisa, assinando o termo de consentimento.

\section{Coleta de dados}

Os dados foram colhidos através da realização deentrevistas face a face, real izadas preferencialmente no domicílio, entre março e julho de 2006, mediante aplicação de questionário estruturado. As entrevistas foram conduzidas por uma equipede alunas do 70 e do 8 o períodos da Faculdade de Serviço Social da Universidade Federal Fluminense (UFF), após treinamento visando à padronização da abordagem e aplicação correta do questionário, supervisionadas por um dos autores (PCAJ). Os idosos foram entrevistados de forma individualizada, após contato inicial com uma entrevistadora, preservando-se a pri- 
vacidade das entrevistadas e mantendo-se sua identidade sob sigilo.

Visando reduzir as taxas de não resposta, aumentar a aceitabilidade do instrumento e a validade das informações como um todo, al guns cuidados especiais na condução das entrevistas foram tomados. M ontou-se um esquema de supervisão de campo que previa um apoio para o entrevistado e a entrevistadora nos casos agudos deviolência ou em situações derisco, e o encaminhamento e acolhimento pelo módulo de Saúde da Família da área dos idosos que fossem identificados como vítimas de violência ou em situações de vulnerabilidade. A utilização de entrevistadoras com formação na graduação em serviço social, assim como o treinamento contínuo, capacitando para uma abordagem acolhedora e facilitadora da revelação das informações mais íntimas, foi outra estratégia empregada para aumentar a qualidade das informações da pesquisa.

Instrumentos de aferição da pesquisa (avaliação)

0 instrumento de coleta de dados foi composto por módulos de questionários já testados e validados em estudos anteriores realizados no Brasil e módulos elaborados especificamente para a pesquisa. Sua estruturação baseou-se na avaliação multidimensional do idoso. 0 questionário final apresentou seis módulos: sociodemográfico, utilização de serviços de saúde, saúde física, saúde mental, capacidade funcional, além do questionário de caracterização da violência.

Os primeiros módulos do questionário foram compostos por questões relativas à caracterização sociodemográfica do entrevistado, deixando as questões potencialmente constrangedoras para momento posterior, quando já se tinha estabelecido uma relação positiva entre 0 entrevistador e os idosos entrevistados no domicílio. 0 primeiro módulo continha dados deidentificação que compreendiam características demográficas básicas, tais como idade, número de filhos, coabitação, naturalidade, cor/raça e estado civil, além de informações sobre indicadores socioeconômicos: escolaridade, ocupação e posse de utensílios domésticos.

Para a captação das variáveis demográficas, utilizou-se o questionário da Pesquisa Nacional por Amostragem de Domicílio ${ }^{17}$. Para a coleta das informações sobre a saúde física do idoso, foram utilizadas as questões do instrumento empregado no inquérito domiciliar sobre comportamento de risco e morbidade referida de doenças eagravos não transmissívei ${ }^{18}$. Além disso, houve a inclusão de patologias que não constavam da versão original, por terem alta prevalência em idosos do nosso meio e, possivelmente, estarem associadas à violência.

Para caracterização dos idosos de acordo com o seu grau de vulnerabilidade decorrente de suas comorbidades, foram criadas duas variáveis (vulnerabilidade 1 e2) que agregavam os indivíduos que apresentavam as doenças: depressão e/ou incontinência urinária e/ou diabetes e/ ou reumatismo; e as doenças: depressão e/ou incontinência urinaria ou fecal eou demência/problemas de memória e ou/ reumatismo, respectivamente.

A saúde mental foi avaliada através do M iniexame do Estado M ental (M EEM $)^{19}$. O M EEM é composto por trinta itens, com subtestes que avaliam orientação espaço-temporal, memória imediata, evocação, memória procedimento elinguagem. Usado isoladamente ou incorporado a instrumentos mais amplos, permite a avaliação da função cognitiva e rastreamento de quadros demenciais ${ }^{20,21}$. Tem sido utilizado em ambientes clínicos, para a detecção de declínio cognitivo, para o seguimento de quadros demenciais e no monitoramento de resposta ao tratamento. Desde sua criação, suas características psicométricas têm sido avaliadas, tanto na sua versão original quanto pelas inúmeras traduções/adaptações para várias línguas e países. Para melhorar a qualidade das informações, nas situações em que o idoso apresentava escore do M EEM inferior a 14 (idosos com menos de quatro anos de estudo) e 18 pontos (idosos com quatro anos ou mais) , a entrevista era realizada com seu principal cuidador. Seguindo orientação deAlmeida ${ }^{20}$, considerou-secomo um caso suspeito de demência aqueles indivíduos que tivessem M EEM < 19 (idosos com menos de quatro anos de estudo) e $<23$ pontos (os demais).

Para avaliação da capacidade funcional do idoso, utilizou-se o H ealth Assessment Questionnaire (HAQ), em razão de suas excelentes propriedades psicométricas ${ }^{22}$. Foi originalmente desenhado para a avaliação clínica de pacientes adultos com artropatias, mas tem sido utilizado numa ampla gama deambientes de pesquisa para avaliação de cuidado. A dimensão de incapacidade é composta por vinte questões que abordam o grau de dificuldade dos idosos em desenvolver atividades da vida diária na última semana, a saber: vestir-se e arrumar-se, levantar-se, alimentar-se, caminhar, realizar atividades de higiene, alcançar e realizar atividades fora do domicílio. Cada item tem um escore dequatro pon- 
tos, variando de "sem dificuldade alguma" até "incapaz de realizar". 0 escore mais alto em cada um dos oito componentes é somado para formar um total (0-24). Este total é dividido por oito para dar um escore de $0-3$, que representa 0 índice de incapacidade funcional do idoso.

Para uma apreciação inicial, foram considerados autossuficientes os idosos que apresentavam escores de 0 a 0,5; idosos que tinham dificuldades leves com pontuação de 0,5 a 1,25, capazes de realizar suas AVDS, e indivíduos com dificuldades importantes, possuindo incapacidade grave, sendo ainda autossuficientes aqueles com pontuação variando de 1,25-2,0. 0 paciente com escores de 2,0 a 3,0 pode ser classificado como possuindo incapacidade grave ${ }^{23}$.

Para avaliar a suspeita de uso abusivo de álcool pelo idoso do sexo masculino, foi utilizado 0 instrumento CAGE (Cut-down; Annoyed; Guilty \& Eye-opener, considerando-se um resultado positivo para ele quando duas ou mais perguntas obtêm a resposta afirmativa ${ }^{24}$. Para a idosa ou cuidadora, utilizou-sea versão nacional do instrumento TWEAK ${ }^{25-26}$, considerando-se como um caso suspeito de "uso inadequado" de álcool as mulheres que responderam positivamente a duas ou mais questões.

Para identificação da violência contra o idoso, utilizou-se a versão nacional das Escalas Táticas de Conflitos (Conflict Tactics Scales CTS1) ${ }^{27,28}$. Este instrumento é composto por 19 itens que abordam a utilização de diferentes táticas para a resolução de conflitos entre pessoas íntimas, a saber: argumentação, agressão verbal, violência física não abusiva eviolência física abusiva. Foram considerados caso positivo de violência psicológica efísica os idosos que referiram ter sido vítimas de pelo menos um dos itens que compõem as subescal as aplicadas nos idosos residentes na I lha da Conceição.

\section{Processamento/análise dos dados}

A entrada de dados e o controle de qualidade foram realizados utilizando-se o programa Epi Info ${ }^{\mathrm{TM}}$ 3.3.2. ${ }^{14}$. O programa STATA v.9.2 foi usado para o processamento e análise de dados. Estimaram-se a prevalência da violência física epsicológica e seus respectivos interval os de confiança a $95 \%$ na população total entrevistada e nos vários subgrupos criados a partir das características sociodemográficas estudadas. Para avaliação da heterogeneidade das proporções nos subgrupos, utilizou-se o teste Qui-quadrado. Assumiu-se uma distribuição binomial para a estimação dos intervalos de confiança.
A pesquisa foi autorizada pela Secretaria M unicipal deSaúde do município eaprovada pelo Núcleo deEducação Permanente(NEPE) do Polo de Capacitação em Saúde da Família da Fundação M unicipal de Saúde de N iterói, uma vez que respeitou os princípios éticos contidos na Declaração deH elsinque ea Resolução n¹96/1996 do Conselho Nacional deÉtica em Pesquisa, obedecendo às normas e diretrizes regulamentadoras das pesquisas envolvendo seres humanos, neste caso os idosos.

\section{Resultados}

Foram entrevistados 343 idosos ( $38,2 \%$ homens e $62,1 \%$ mulheres). Como pode ser visto na Tabela 1, a maioria dos idosos entrevistados apresentava entre 60 e 70 anos. Em relação à cor da pele, a maior parte dos respondentes se identificou com a cor negra/mulato. Com relação à situação conjugal, grande parte dos entrevistados referiu ser casado, havendo também um contingente razoável de viúvos.

Tabela 1. Características sociodemográficas da população estudada, PMF, Ilha da Conceição, Niterói, RJ, em 2006.

\begin{tabular}{lrr}
\hline Características sociodemográficas & $\mathrm{n}$ & Prevalência (IC 95\%) \\
\hline Sexo & & \\
$\quad$ M asculino & 130 & $37,9(32,7-43,1)$ \\
$\quad$ Feminino & 213 & $62,1(56,9-67,3)$ \\
Faixa etária & 194 & $56,6(51,3-61,8)$ \\
$60-70$ & 123 & $35,9(30,8-41,0)$ \\
$71-80$ & 26 & $7,6(4,8-10,4)$ \\
$\quad>80$ & & \\
Cor/Raça & 165 & $48,1(42,8-53,4)$ \\
$\quad$ Branca & 178 & $51,9(46,6-57,2)$ \\
$\quad$ Negro \& M ulatos & & \\
Estado Civil & 171 & $49,8(44,5-55,2)$ \\
$\quad$ Casado & 111 & $32,4(27,4-37,3)$ \\
$\quad$ Viúvo & 61 & $17,8(13,7-21,9)$ \\
$\quad$ Outros & & \\
Escolaridade & 262 & $76,4(71,9-80,9)$ \\
$\quad$ Até 4 série & 54 & $15,7(11,9-19.6)$ \\
$\quad$ De 5 a 8 série & 27 & $7,8(5,0-10,7)$ \\
$\quad$ Maior que 8 série & & \\
Numero de moradores do domicílio & 76 & $22,2(17,7-26,6)$ \\
$\quad$ Mora sozinho & 107 & $30,9(26,0-35,8)$ \\
$\quad$ M ora com uma outra pessoa & 160 & $46,6(41,3-51,9)$ \\
$\quad$ Mora com duas ou mais pessoas & & \\
Profissão & 115 & $33,5(28,5-38,6)$ \\
$\quad$ Do lar & 228 & $66,5(61,5-71,5)$ \\
$\quad$ Outros & & \\
Situação previdenciária & 199 & $60,7(55,4-66,0)$ \\
$\quad$ Aposentado & 129 & $39,3(34,1-44,6)$ \\
$\quad$ Não aposentado & & \\
\hline
\end{tabular}


A amostra congregou idosos de baixa escolaridade, na medida em que $76,4 \%$ dos respondentes relataram ter tido quatro anos ou menos de estudo, enquanto apenas cerca de $8,0 \%$ referi-

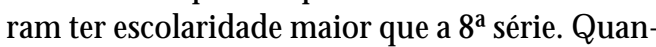
to à composição doméstica, observa-seum grande contingente de idosos morando sozinho ou com apenas mais uma pessoa. A maioria refere ter uma profissão, mas ser atualmente beneficiário da Previdência Social.

A Tabela 2 apresenta a situação de saúde dos idosos entrevistados. A grande maioria refereter a presença de pelo menos uma patologia, sendo a hipertensão arterial a principal morbidade referida (72,6\%; IC 95\%: 67,7 - 77,4). Cercade 10\% dos idosos do sexo masculino foram considerados positivos para o uso inadequado de álcool. No entanto, nenhuma das idosas foi identificada com tal situação.

Constatou-se que cerca de $20 \%$ dos idosos foram considerados suspeitos de um quadro de demência. Com relação à capacidade funcional, observou-se que a maioria dos idosos entrevistados foi considerada autossuficiente, situação confirmada pela baixa prevalência de idosos que necessitavam de cuidadores para ajudá-los a desempenhar as tarefas do dia a dia.

A Tabela 3 apresenta a situação da população deestudo em relação à prevalência das diferentes formas de violência doméstica. Como pode ser observado, $43,2 \%$ relataram ter sofrido pelo menos um episódio de violência psicológica no ano anterior à entrevista. A violência física de qualquer gravidade foi relatada por cerca de $10 \%$ dos entrevistados, enquanto $6,1 \%$ dos idosos referiram a ocorrência de violência física grave nesse período.

A Tabela 4 apresenta a prevalência das diversas formas de violência nos diferentes subgrupos populacionais. Como pode ser observado, de modo geral a prevalência das diversas modalidades de violência foi maior entre os mais no-

Tabela 2. M orbidade referida e agravos identificados na população estudada, PM F, I lha da Conceição, Niterói, RJ, em 2006.

\begin{tabular}{|c|c|c|}
\hline Morbidades & $\mathrm{n}$ & Prevalência (IC 95\%) \\
\hline \multicolumn{3}{|l|}{ M orbidades referidas } \\
\hline Hipertensão arterial & 238 & $72,6(67,7-77,4)$ \\
\hline Reumatismo & 137 & $44,5(38,9-50,1)$ \\
\hline Doença cardíaca & 68 & $22,1(17,4-26,7)$ \\
\hline Diabetes mellitus & 57 & $18,6(14,2-22,9)$ \\
\hline Demência & 52 & $17,1(12,8-21,3)$ \\
\hline Depressão & 49 & $16,2(12,0-20,4)$ \\
\hline Acidente vascular cerebral (AVC) & 24 & $7,9(4,9-11,0)$ \\
\hline Incontinência urinaria/fecal & 23 & $7,6(4,6-10,6)$ \\
\hline DPOC (enfisema pulmonar) & 10 & $3,3(1,3-5,3)$ \\
\hline \multicolumn{3}{|l|}{ Concomitância dos agravos } \\
\hline Relato de pelo menos uma das patologias acima & 292 & $85,1(81,3-88,9)$ \\
\hline Relato de três ou mais das patologias acima & 107 & $31,2(26,3-36,1)$ \\
\hline \multicolumn{3}{|l|}{ Concomitância de patologias específicas a } \\
\hline Vulnerabilidade 1 & 175 & $51,0(45,7-56,3)$ \\
\hline Vulnerabilidade 2 & 190 & $55,4(50,1-60,7)$ \\
\hline Suspeição de abuso de álcool b & 39 & $11,4(8,0-14,7)$ \\
\hline Suspeita de demência (MEEM positivo) ${ }^{c}$ & 68 & $19,8(15,6-24,1)$ \\
\hline Presença de cuidador & 72 & $21,0(16,7-25,3)$ \\
\hline \multicolumn{3}{|l|}{ Suspeita de incapacidade funcional d } \\
\hline Autossuficientes & 245 & $71,4(66,6-76,2)$ \\
\hline Dificuldades leves & 54 & $15,7(11,9-19,6)$ \\
\hline Dificuldades importantes & 27 & $7,8(5,0-10.7)$ \\
\hline Incapacidade & 17 & $5,0(2,6-7,3)$ \\
\hline
\end{tabular}

Legenda: a) Vulnerabilidade 1: refere depressão e/ou incontinência urinária e/ou diabetes e/ou reumatismo; Vulnerabilidade2: refere depressão e/ou incontinência urinária ou fecal e/ou demência/problema dememória e/ou reumatismo (artrose) :

b) Consideraram-se casos suspeitos os idosos que tivessem dois ou mais itens positivos no instrumento CAGE; c) Consideraram-se positivos os idosos que tivessem M EEM < 19 para aqueles com menos de quatro anos de estudo e < do que 23 pontos entre aqueles com quatro anos ou mais de estudo; d) Consideraram-se autossuficientes os que apresentavam escores de 0 a 0,5 com dificuldade leves com pontuação de 0,5 a 1,25, capazes de real izar suas AVDS e idosos com alterações importantes possuindo incapacidade grave, porém ainda autossuficientes, com pontuação variando de 1,25-2,0. 
vos, com maior escolaridade, entre os que apre sentam pelo menos uma das patologias que caracterizam o idoso com tendo uma vulnerabilidade (depressão e/ou incontinência urinária/fecal e/ou diabetes e/ou reumatismo e/ou diabetes) eentre os quemoram com maior número de indivíduos. Percebem-se algumas particularidades ao se apreciar o perfil de ocorrência de cada
Tabela 3. Prevalência das diferentes formas de violência doméstica contra os idosos, PMF, Ilha da Conceição, Niterói, RJ, em 2006.

\begin{tabular}{lrr}
\hline Tipos de violência & \multicolumn{1}{c}{$n$} & Prevalência (IC 95\%) \\
\hline Violência psicológica & 148 & $43,2(37,9-48,4)$ \\
Violência física grave & 21 & $6,1(3,6-8,7)$ \\
Violência física total & 33 & $9,6(6,5-12,8)$ \\
\hline
\end{tabular}

Tabela 4. Prevalência de violência doméstica psicológica e física em diferentes subgrupos da população adscrita ao PSF, PM F, Ilha da Conceição, Niterói, RJ em 2006.

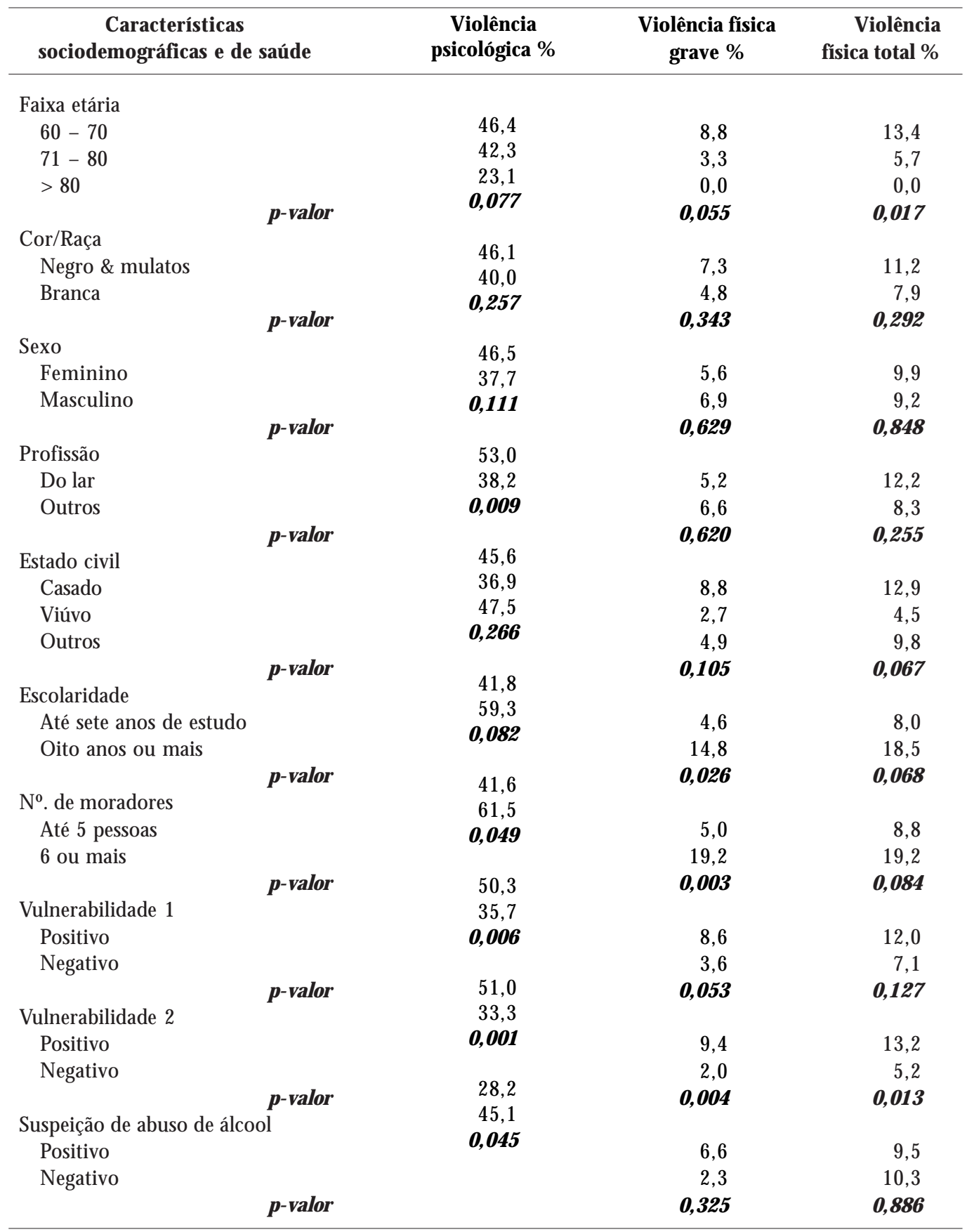


tipo de violência isoladamente. Enquanto é nítida a maior prevalência de violência psicológica entre os que se referiram como "do lar" no momento da entrevista e entre os que não foram identificados como suspeitos de abuso de álcool, não se pode afirmar que a violência física seja mais frequente nesses subgrupos. Já ao se avaliarem as diferenças entre as prevalências de violência física total e grave, ainda que com certa ressalva devido à menor significância estatística, é possível perceber que entre os casados o problema parece ser bem mais freqüente.

\section{Discussão}

0 envelhecimento acelerado da população brasileira vem sendo apontado por diversos autores $^{1,2,29,30}$. N esse contexto, a promoção de um envelhecimento saudável se apresenta como um dos grandes desafios da sociedade em geral e particularmente do setor saúde neste século que se inicia. Dentre os grandes empecilhos à saúde do idoso, a violência vem sendo cada vez mais colocada em pauta, particularmente a que acorre no âmbito familiar, já que, segundo alguns autores, $90 \%$ dos casos de maus-tratos e negligência contra as pessoas acima de 60 anos ocorrem nos lares ou em instituições asilares, ${ }^{6,831,32}$.

No Brasil eparticularmente no estado do Rio de Janeiro, os estudos sobre o tema são ainda muito incipientes, impedindo estimativas mais concretas sobre a magnitude do problema. No melhor do conhecimento dos autores, este é o primeiro inquérito populacional realizado no estado que estima a prevalência de violência doméstica contra o idoso não institucionalizado. Dessa forma, apesar deter sido realizado em uma área específica de N iterói, espera-se que os resultados da pesquisa contribuam para a divulgação do tema e ampliem a discussão sobre como intervir visando ao seu enfrentamento.

0 perfil sociodemográfico da população entrevistada é, de modo geral, semelhante ao de outros estudos que envolveram as parcelas menos favorecidas dos idosos brasileiros ${ }^{1,2,33}$. A necessidade de restrição da capacidade de generalização dos achados às comunidades de alto risco social eeconômico é reforçada quando se focalizam suas características em termos de escolaridade. Ao se analisar o perfil educacional da população estudada, observa-se que esta tem um grau de escolaridade que parece ser bastante inferior ao estimado para o Brasil como um todo e mesmo para o conjunto de idosos da cidade de
Niterói. Considerando todo o país, os dados divulgados pelo Instituto Brasileiro de Geografia e Estatística (IBGE) relativos ao ano de 1996 estimavam que aproximadamente $41 \%$ das mulheres idosas e $37 \%$ dos homens eram analfabetos, parcelas provavelmente bem menores do que as estimadas para esta amostra se considerarmos a proporção de idosos com menos de 4 anos de escolaridade $^{34}$. A menor escolaridade da amostra vis-à-vis ao conjunto dos idosos da cidade fica evidente ao se compararem os achados do estudo aos divulgados pelo IBGE para o ano de 2004, que estimavam uma média de anos de estudos da população maior de 60 anos de 8,2 anos, bastante superior à estimada para a população do estudo ${ }^{14}$.

O perfil de saúde dos idosos também é bastante peculiar, na medida em que indica uma população que tem grande autonomia, apesar deacumular diferentes morbidades eter uma alta prevalência de casos suspeitos de demência. A manutenção da autonomia por boa parte dos idosos também édescrita por outros autores que vêm trabalhando com a população dessa faixa etária. Independentemente de suas comorbidades, a maior parte dos idosos tem sua autonomia e independência preservadas ${ }^{1,2,235,36}$.

A alta prevalência de casos suspeitos de demência também merece discussão. Infelizmente, os estudos populacionais sobre o tema são escassos tanto no Brasil como em outros países em desenvolvimento, não havendo ainda estimativas precisas da sua incidência e prevalência ${ }^{37,38}$. Diantedessa limitação, atualmente a prevalência de pessoas com demência nos países em desenvolvimento é estimada a partir dos parâmetros obtidos nos países desenvolvidos, girando em torno de 3\%. Dessa forma, é preciso ressaltar que o instrumento utilizado no estudo é apenas uma ferramenta de rastreamento e não diagnóstica. Somente a partir do encaminhamento dos idosos positivos ao PM F para uma investigação diagnóstica mais sensível eespecífica seria possível estimar a real prevalência do problema.

A heterogeneidade existente no que diz respeito aos conceitos relativos à violência impõe que qualquer comparação de resultados entre estudos deva ser feita com cautela. As diferentes definições conceituais eoperacionais, bem como os instrumentos de coleta de dados utilizados para a identificação das situações de violência, também tornam complexa a tarefa de comparação externa. Ainda assim, vale confrontar as prevalências encontradas neste estudo com aquelas de outros países e regiões brasileiras, desde que 
consideradas as particularidades metodológicas e as diferenças culturais.

A prevalência de diversos tipos de violência variou no estudo entre $43,2 \%$ (violência psicológica) a 6,1\% (violência física grave). Tais estimativas são bastante semelhantes às encontradas em alguns estudos realizados nos Estados Unidos e no Canadá, que estimam uma prevalência de maus-tratos físicos em $10 \%$ para toda a população idosa ${ }^{39-41}$. M ostra-se, no entanto, bem inferior a outras pesquisas norte-americanas, que apontam prevalências de $36 \%$ e $81 \%$ de abuso físico e abuso psicológico no ano anterior à entrevista, respectivamente ${ }^{42}$. Em outra pesquisa recente, estimou-se que mais de dois milhões de idosos sejam anualmente vítimas de abuso nos Estados Unidos ${ }^{43}$

Como descrito, a maior prevalência de abuso psicológico vis-à-vis aos demais encontrada na população de estudo também é relatada em outros estudos ${ }^{22-44}$. Diante da alta magnitude do problema e das consequências nefastas dessetipo de violência à saúde do idoso, vários autores têm enfatizado a necessidade de encarar o problema como uma grave forma de agressão, que necessita de ações de enfrentamento imediato nesse grupo populacional.

A frequência da violência física nos diferentes subgrupos populacionais também merece discussão. Os resultados deste estudo corroboram alguns achados de estudos anteriores e contradizem outros. De acordo com a literatura sobre o tema, esperava-se que as diversas formas de violência fossem mais frequentes entreos idosos mais velhos, com menor escolaridade, que morassem em lares com maior número de indivíduos, casados, com comprometimento da saúde física e mental, com al gum grau de incapacidade funcional equefizessem uso abusivo do álcoo|5,9,45,46. De fato, entre os idosos entrevistados, de modo ge ral, a prevalência do problema foi mais elevada entre os de escolaridade baixa, com maior número de filhos e entre os que apresentavam certas patologias. Porém, não foram observadas diferenças estatisticamentesignificativas entreas prevalências das várias formas de violência nos demais subgrupos estudados.

Com relação à saúde física e mental, vários autores vêm indicando que os idosos com história de maus-tratos apresentam maior prevalência de demência, depressão e problemas reumatológicos do que aqueles quenão foram vitimizados $^{47,48}$. Em estudo brasileiro realizado no estado de São Paulo, a associação entre maus-tratos e essas patologias também foi apontada ${ }^{49}$. Na pre- sente pesquisa, por questões de eficiência estatística, optou-se por estudar essas patologias em conjunto. De acordo com os achados, parece que aqui a situação não é diferente, já que a violência (de qualquer gravidade) esteve associada ao relato de pelo menos uma dessas morbidades.

Dentre as várias hipóteses postuladas para justificar a maior ocorrência da violência contra os idosos entre aqueles com certas patologias, a perda da autonomia física e cognitiva - que gera a necessidade de outra pessoa para real ização das atividades diárias e instrumentais, criando, consequentemente, subordinação - parece ter lugar de destaque entre os pesquisadores ${ }^{8,50,51}$. Já outros autores sugerem que os agravos sejam decorrentes da violência sofrida e não fatores propensores do evento ${ }^{52}$. Neste estudo não se evidenciou maior prevalência das várias formas de violência entre os idosos com maior dependência, o que questiona ambas as hipóteses. Porém, devido à restrição de um estudo transversal, cuja análise de dados explorou apenas a distribuição dos eventos de acordo com cada uma das variáveis de interesse isoladamente, não se pode descartar a possibilidade de a semelhança entre as prevalências do problema nos subgrupos serem decorrentes de distribuições desiguais de outras variáveis envolvidas no processo. Para compreender o papel da dependência funcional na ocorrência de violências aos idosos, seria interessante que estudos futuros, utilizando os procedimentos indicados para se avaliarem associações causais, fossem desenvolvidos.

Outro ponto que merece discussão diz respeito às prevalências das várias formas de violência entre os idosos com suspeita de abuso de álcool. Vários estudos ressaltam a forte associação entre maus-tratos aos velhos e dependência química do idoso ou de seu cuidador $5,39,53$. No presente estudo, não só não se observou maior frequência da violência física entreosidosos suspeitos de abuso de álcool, como a violência psicológica foi mais frequente exatamente entre os que não foram identificados como tal. A pesar de não se ter informações suficientes para se tirar conclusões sobre esse achado, é possível postular al gumas hipóteses explicativas para o ocorrido. U ma das possibilidades aventadas diz respeito ao grau de adequação dos instrumentos de suspeição de abuso de álcool utilizados no estudo. Tanto o CAGE como o TWEAK foram originalmentepropostos parautilização entrehomens e mulheres jovens e adultas, não sendo especificamente desenhados para a detecção do uso inadequado de bebidas alcoólicas por idosos. Tais 
questionários são formados por itens que, em sua maioria, se baseiam na desaprovação social e do próprio indivíduo sobre seu consumo de bebidas alcoólicas. Em razão do senso comum de que os indivíduos idosos não devem consumir qual quer quantidade de bebida alcoólica devido às suas comorbidades e ingesta de medicamentos, épossível quemuitos dosidosos tenham respondido positivamente às questões sem que isto corresponda a uma situação de fato abusiva. Dessa forma, haveria uma má classificação dos idosos positivos devido à baixa especificidade do instrumento, o que reduz a qualidade da informação como um todo, inviabilizando uma apreciação mais robusta.

Outras limitações metodológicas também devem ser apontadas e debatidas. Apesar de as estimativas de vitimização por maus-tratos serem el evadas, o quese percebeno contexto clínico é que a situação frequentemente não é revelada pelo idoso, seus familiares ou cuidadores. A pesar de ser expressivo o número de idosos que sofrem todos os tipos de maus-tratos, na maior parte das vezes estes se calam, por medo de represália da própria família e/ou cuidador, ou mesmo por desinformação. Dessa forma, é possível imaginar que, apesar do esforço realizado com vistas à garantia da qualidade das informações na detecção das situações de violência, as estimativas apresentadas possam estar subestimadas.

Visando diminuir a limitação, optou-se pela formação de uma equipe de entrevistadoras acoIhedora ebem treinada, além da utilização deum questionário multidimensional, o que pode ter criado uma atmosfera de confiança entre entrevistador-entrevistado, facilitando o relato das experiências pessoais mais íntimas e a revelação de situações de violência que, de outra forma, passariam despercebidas. Outro ponto importante éque, embora setenhautilizado a CTS, que é um instrumento estruturado já validado em outros contextos para a identificação das situações de violência, foram encontrados poucos estudos que a tenham usado especificamente em idosos. Deconcepção mais antiga, a CTS foi, originalmente, criada para avaliar violência íntima em indivíduos maisjovens. Não surpreendeque, mesmo sendo bastante utilizada no Brasil, sua adaptação tenha-se dado em ambiente de violência focalizando faixas etárias aquém dos 60 $a_{0}{ }^{52}$. N esse contexto, não se sabe exatamente a validade do instrumento na detecção das situações de violênciafísica entreindivíduos dessa faixa etária, o que faz indagar se as estimativas da pesquisa não estariam subestimadas. Ademais, por ter sido desenvolvido focalizando outras faixas etárias, a utilização do instrumento não permitiu que se avaliasse a ocorrência de situações denegligência, abandono eabuso econômico, que vêm sendo enfatizados na literatura internacional como importantes facetas da violência doméstica ao idoso.

Outra lacuna a ser preenchida em estudos futuros diz respeito à coleta de informações com outrosindivíduos que convivam com o idoso em seu ambiente doméstico. Estudos internacionais vêm chamando a atenção para a necessidade de incorporação da entrevista aos cuidadores na detecção de situações de risco potencial para a violência ao idos5 5,645. A entrevista de acompanhantes presentes na maior parte das vezes no convívio com o idoso pode complementar a informação conseguida através da avaliação das próprias vítimas em potencial ${ }^{55}$. Infelizmente, por questões operacionais, exceto em situações graves de déficits cognitivos, as informações sobre violência foram apreendidas apenas através da entrevista com o próprio idoso, o que também pode ter diminuído a sensibilidade do processo de detecção das situações de vitimização da violência.

Diante da relevância do problema e da escassez de estudos em nosso meio, torna-se fundamental a ampliação dos programas de investigação nessa área, visando ao detalhamento das características e contextos deocorrência do problema para o planejamento de ações efetivas para seu enfrentamento. No âmbito clínico, énítida e urgente a necessi dade da inclusão dessa temática nas escolas de formação de profissionais de saúde e a discussão do tema nos serviços de saúde. 0 abuso em idosos vem em múltiplas formas, cujas consequências frequentemente se sobrepõem e se confundem com sinais e sintomas relacionados a diversas patologias prevalentes nessa faixa etária. Dessa forma, é fundamental um olhar aguçado e sistematizado que permita levantar a suspeita, confirmar 0 abuso nesse grupo de idosos.

Na perspectiva dos profissionais do Programa Saúde da Família, para realizar uma investigação, um diagnóstico médico de abuso em idosos é um marco exitoso, pois qualquer possibilidade de simplificar, fortalecer ou otimizar o processo deidentificação édesejável. Linguagem compartilhada com os velhos acerca de suas vivências, respeitando a complexidade da situação, valorizando e respeitando os sofrimentos decorrentes da violência, pareceser um bom canal para uma atuação baseada na promoção da saúde e prevenção da violência. Além das ações de pro- 
moção e prevenção primária, a Estratégia Saúde da Família deve ter lugar de destaque nas propostas que visem à atuação dos serviços de saúde, tanto na detecção precoce do problema como na interrupção de uma situação já instalada. A participação de agentes de saúde na formação das equipes também favorece ações que visem à identificação de idosos em situação de violência, na medida em que os agentes realizam visitas domiciliares com frequência, podendo ter acesso às situações que não aparecem nas consultas de rotina nas unidades de saúde. Ademais, sua origem na comunidade permite, muitas vezes, a suspeição de situações que passariam despercebidas pelos serviços.

A opção pela abordagem familiar em contraposição à individual é outro trunfo das equipes de Saúde da Família, tanto na prevenção como na detecção precoce da violência ao idoso. $A$ inclusão dos familiares cuidadores/acompanhantes nas ações desaúde facilita o processo, já quea saúde e as circunstâncias de vida dos cuidadores estão, com frequência, intimamenteligadas àquelas das vítimas. Destaforma, a experiência clínica sugereque reconhecimento da díadecuidadoridoso e a interven ção direta nesse relacionamento oferecem possibilidades concretas de se que brar um eventual ciclo de violência em curso ${ }^{55}$. A legislação existente, como Estatuto do Idoso, Política Nacional de Redução deAcidentes eViolências do M inistério da Saúde, Plano deAção de Enfrentamento daViolência contra a Pessoal dosa, mostra a abertura do Brasil para a busca de soluções. Para que a violência contra a velhice desapareça, é necessário o engajamento de toda a sociedade, que precisa reconhecer a importância do tema e sua magnitude.

\section{Conclusão}

Os resultados deste estudo justificam o desenvolvimento de novas pesquisas sobre o tema, como também a necessidade de se discutirem políticas públicas, responsabilidade social e dignidade no envel hecimento, para quevalha a pena viver mais, num contexto em queécada vez maior a expectativa de vida dos idosos. Dessa maneira, cabe agora conscientizar a sociedade da necessidade de se estabelecerem ações que reprimam a violência ao idoso. Outra questão central diz respeito à necessi dade dese aperfeiçoar ainda mais os profissionais de saúde que assistem essa significativa parcela da população.

Os profissionais da área da saúde tendem a subestimar a importância da violência familiar, sendo ainda muito precária a deteç̧ão de casos em serviços de atenção primária, como o Programa M édico de Família, quando se leva em conta a elevada frequência do evento e as oportunidades das equipes em suspeitar queesteesteja ocorrendo. Do mesmo modo, os serviços de saúde têm o dever de constituir-se como um local de acolhimento e elaboração de projetos de apoio contra a violência. Infelizmente, ainda são poucos os serviços preparados e organizados para atender aos casos de violência ao idoso.

A complexidade que envolve a questão da violência ao idoso, no âmbito do Programa M édico de Família, exige ações capazes de dar conta das inúmeras demandas apresentadas, na perspectiva de atenção integral à saúde do idoso. 


\section{Referências}

1. Ramos LR. Fatores determinantes do envelhecimento saudável em idosos residentes em centro urbano: Projeto Epidoso. Cad de Saúde Pública 2003;19:793-798.

2. Kalache A, Veras RP, Ramos LR.O envelhecimento da população mundial: um desafio novo. Rev Saúde Publ. 1987; 21(3):200-210.

3. M inayo, MCS. Violência sob o olhar da saúde. Rio de Janeiro: Editora Fiocruz; 2002.

4. Machado L, Queiroz ZV. Negligência e maus-tratos. In: Freitas, EV de, Py L, Neri A L, Cançado FAX, Gorzoni ML, Rocha SM, organizadores. Tratado de geriatria e gerontologia. Rio de Janeiro: Guanabara Koogan; 2002. p. 1152-1159.

5. M oya AB, Gutierre JB. M alos tratos a personas mayores: guia de actuación. Madrid: Ministério deTrabajo y Asuntos Sociales; 2005.

6. Krug EG, Dahlberg LL, M ercy JÁ, Zwi AB, Lozano $R$, editors. Missing voices: views of $H$ oover persons on elder abuse. Genebra: WHO; 2002.

7. Brasil. M inistério da Saúde. Programa Saúde da Família. Violência intrafamiliar: orientações para a prática em serviço. Brasília: Ministério da Saúde; 2002. (Cadernos de Atenção Básica, 8).

8. Pillermer KA, Moore DW. Abuse of patients in nursing homes: Findings from a survey of staff. Gerontologist 1989; 3:314-320.

9. Brasil. Ministério da Saúde. Política Nacional de Redução da M ortalidade por Acidentes e Violências. Brasília: Ministério da Saúde; 2002.

10. World Health Organization. Violence against women. Geneva; 1997.

11. Pillermer KA, Prescott D. Psychological effeets of elder abuse, a research not. Jornal of elder abuse and neglect 1989; 1(1):379-392.

12. Apratto PC. Refletindo sobre a metodologia do Programa M édico de Família. Niterói: Fundação M unicipal de Saúde/Programa M édico de Família; 1998.

13. Instituto Brasileiro de Geografia e Estatística (IBGE). Contagem da população. 1996. [acessado 2010 ago 25]. Disponível em: http://www.ibge.gov.br/home/ estatistica/populacao/contagem/default.shtm

14. Departamento de Informática do SUS/Sistema de Informação da Atenção Básica (Datasus/Siab). 2006. [acessado 2006 dez 2]. Disponível em: www.datasus. gov.br/idb2006/

15. Espíndola CR, Blay SL. Prevalência de maus tratos na terceira idade: revisão sistemática. Rev Saude Publica 2007; 41(2):301-305.

16. Epi Info [program de computador] Versão 2002. [acessado 2006 dez 2]. Disponível em: http://www. cdc.gov/epiinfo/ei2002.htm
17. Instituto Brasileiro de Geografia e Estatística (IBGE). Pesquisa Nacional por Amostra de Domicílios (PNAD): síntese de indicadores. Rio de Janeiro: IBGE; 2004.

18. Instituto Nacional de Câncer (Inca). Comportamento de risco e morbidade referidas de doenças e agravos não transmissíveis. [acessado $2006 \mathrm{dez} 2$ ]. Disponível em: http://www.inca.gov.br/inquerito/

19. Folstein M F, Folstein SE, M cHugh PR. "M ini-mental state". A practical method for grading the cognitive state of patients for the clinician. J Psychiatr Rese 1975; 12:189-198.

20. Almeida OP. M ini-exame do estado mental e o diagnóstico de demência no Brasil. Arq N europsiquiatr 1998; 56:605-612.

21. Brucki SMD, Nitrini R, Caramelli P, Bertolucci PHF, Okamoto IH. Sugestões para o uso do mini-exame do estado mental no Brasil. Arq N europsiquiatr 2003; 61:777-781.

22. Paixao Júnior CM, Reichenheim ME. U ma revisão sobre instrumentos de avaliação do estado funcional do idoso. Cad. Saúde Pública 2005; 21(1):7-19.

23. Shinjo SK, Gonçalves R, Gonçalves CR. M edidas de Avaliação Clínica em Pacientes com Espondilite Anquilosante: Revisão da Literatura. Rev Bras Reumatol 2006; 46(5):340-346.

24. Da Paz Filho GJ, Sato LJ, Tuleski MJ, Takata SY, Ranzi CCC, Saruhashi SY, Spadoni B. Emprego do questionário cage para detecção de transtornos de uso de álcool em pronto-socorro. Rev Ass M ed Brasil 2001; 47(1): 65-69.

25. Russell M, Martier SS, Sokol RJ, Mudar P, Jacobson $\mathrm{S}$, Jacobson J. Detecting risk drinking during pregnancy: a comparison of four screening questionnaires. Am J Public Health 1996; 86:1435-1439.

26. Moraes CL, Viellas EF, Reichenheim ME. Assessing alcohol misuse during pregnancy: evaluating psychometric properties of the CAGE, T-ACE, and TWEAK in a Brazilian setting. J Stud Alcohol 2005; 66:165-173.

27. Straus MA, Hamby SL, Boney-M ccoy S, Sugarman DB. The revised Conflict Tactics Scales (CTS2): Development and preliminary psychometric data. Journal of Family Issues 1996; 17:283-316.

28. Hasselmann MH, Reichenheim M E. Adaptação transcultural da versão em português das "Confl ict Tactics Scales Form R" (CTS-1) usada para aferir violência no casal: equivalências semântica e de mensuração. Cad Saude Publica 2003;19(4):1083-1093.

29. Carvalho JAM, Garcia RA. O envelhecimento da população brasileira: um enfoque demográfico. Cad Saude Publica 2003; 19(3): 725-733. 
30. Camarano A. Os novos idosos brasileiros, muito além dos 60. Rio de Janeiro: IPEA; 2004.

31. Barnett O, M iller-Perrin C, Perrin RD. History and definitions. In: Barnett O, Miller-Perrin C, Perrin RD, editors. Family violence across the lifespan: an introduction. London: Sage Publications; 1997. p. 3-17.

32. Kosberg JI. The abuse of elderly men. Journal of Elder Abuse and Neglect 1998; 9(3):69-88.

33. Veras R. Em busca de uma assistência adequada à saúde do idoso: revisão da literatura e aplicação de um instrumento de detecção precoce e de previsibilidade de agravos. Cad Saude Publica 2003; 19(3):705-715.

34. Instituto Brasileiro de Geografia e Estatística(IBGE). Censo Demográfico de 1991: resultados definitivos. São Paulo: Rio de Janeiro; 1996. (CD-ROM com os microdados da amostra).

35. Veras RP, organizador. Terceira Idade: um envelhecimento digno para o cidadão dofuturo. Rio de Janeiro: UnATI/Relume Dumará;1995.

36. Chaimovicz F. Os idosos brasileiros no século 21. Belo Horizonte: Postgraduate Brasil, 1998.

37. Hendrie HC. Exploration of environmental and ge netic factors for Alzheimer's disease: the value of cross-cultural studies. Current Directions in Psychological Science 2001; 10:98-101.

38. Herrera JRE, Caramelli P, Silveira ASB, Nitrini R. Epidemiological Survey of Dementia in a Communitydwelling Brazilian Population Alz. Dis. Assoc. Disord. 2002; 16:103-108.

39. Chavez N. Violence against the elderly. 2002 April. Disponível em: http:/www.health.org/referrals/ resguides._asp.

40. Kleinschimidt KC. Elder abuse: A review. Annals of Emergency M edicine 1997; 30:463-472.

41. Wolf RS. Maltrato en ancianos. In: Anzola-Perez E, organizadora. Atención de los Ancianos: Un Desafio para los Noventa. Washington, DC: Pan American Health Organization; 1995. p. 35-42.

42. Pillemer KA, Moore DW. Abuse Patients in Nursing Homes: Findings from a Survey of Staff. The Gerontologist 1989; 29:314-320.

43. Swagerty DL, Takahashi PY, Evans JM. Elder M istreatment. Am Fam Physician 1999; 59(10):2804-2808.

44. World report on violence and health. Abuse of the elderly. In: World report on violence and health. Geneva: World Health Organization; 2002. p. 123145.

45. Baumhover LA, Beall SC. Prognosis. In: Baumhover LA, Beall SC, publishing. Abuse, neglect and exploitation of older persons: strategies for assessment and intervention. Baltimore: Health Professions Press; 1996.
46. Pillemer K, Finkelhor D. The prevalence of elder abuse: a random sample survey. Gerontologist 1988; 28(1):51-57.

47. Cooney C, Mortimer A. Elder abuse and dementia: a pilot study. International Journal of Social Psychiatry 1995; 41(4):276-283.

48. Dyer C, Rowe J. Elder abuse. Trauma 1999; 1:163169.

49. Sanches APRA, Lebrão ML, Duarte YAO. Violência contra idosos: uma questão nova? Saúde Soc 2008; 17(3):90-100.

50. Jones CJ, Rikli RE, Beam WC. A 30-s chair-stand test as a measure of lower body strength in community-residing older adults. Res Q Exerc Sport. 1999; 70(2):113-119.

51. Herrera $\mathrm{H}$, Rebato $\mathrm{E}, \mathrm{H}$ ernandez R, HernandezValera Y, Alfonso-Sanchez MA. Relationship between somatotype and blood pressure in a group of institutionalized Venezuelan elders. Gerontology 2004; 50:223-229.

52. Yan E, Tang C. Prevalence and Psychological Impact of Chinese Elder Abuse. Journal of interpersonal violence 2001; 16(11):1158-1174.

53. Barnett O, Miller-Perrin C, Perrin RD. History anddefinitions. In: Barnett O, Miller-Perrin C, Perrin $\mathrm{RD}$, publishing. Family violence across the lifespan:an introduction. London: Sage Publications; 1997. p. 317.

54. Paixão Júnior $\mathrm{CM}$, Reichenheim ME. U ma revisão sobre instrumentos de rastreamento de violência doméstica contra o idoso. Cad Saude Publica 2006; 22(6):1137-1149.

55. Souza AS, Souza AS, M eira EC, Neri IG, Silva JA, Gonçalves LHT. Fatores de risco de maus tratos ao idoso na relação idoso/cuidador em convivência intrafamiliar. Textos sobre Envelhecimento 2004; $7(2): 63-84$.

Artigo apresentado em 27/05/2007

Artigo aprovado em 14/12/2007

Versão final apresentada em 05/02/2008

Este artigo sofreu al terações por solicitação do editor em N ov/2010 conforme ERRATA publicada no Volume 16 Número 1 do periódico. (http://www.scielo.br/pdf/csc/v16n1/v16nla39.pdf) 